\title{
Lubrication
}

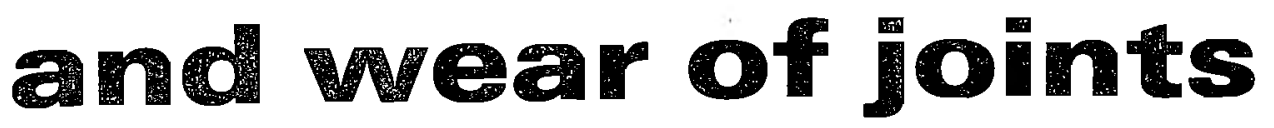

\author{
V. WRIGHT, M.D., F.R.C.P. \\ Professor of Rhelmatology, Rheumatism Research Unit, \\ University Department of Medicine, General Infirmary at \\ Leeds, England.
}

\section{Lubrication and Wear of Joints}

The natural joint is a remarkable bearing. It is expected to operate in the human machine for a period of about 70 years whilst transmitting loads and yet accommodating a wide range of movement. It normally performs these functions with high efficiency, and coefficients of friction as low as 0.002 have been recorded. In engineering situations bearing life is sometimes measured in minutes, often in hours, and sometimes in years, but it is rare indeed for the bearing desigser to be asked to provide a bearing with an estimated life of 70 years in the machine! It is fair to say that the engineer would find it extremely difficult to provide a bearing which would operate within the environment of the body, in the same space as the natural bearing, under the same loads, with the same degree of movement, with similar friction and comparable mean life to the natiral bearing.

The influence of lubrication, friction, wear and good bearing design upon the efficiency and general economic performance of the machinery in our technological society was recognised in 1966 by the introduction of a new word, "tribology', meaning 'the science and technology of interacting surfaces in relative motion'. At the University of Leeds we have formed a Bioengineering Group for the Study of Human Joints which combines the expertise of rheumatologists, orthopaedic surgeons, tribologists, biochemists, polymer scientists, metallurgists, physical educationists and zoologists ir an effort to understand the remarkable characteristics of healthy joints and, perhaps more importintly, the reasons why some human bearings wear out more rapidly than others.

Engineers tend to classify forms of lubrication into a few well-known physical and chemical mechanisms, and a good deal of progress has now been mude towards an understanding of the ferformance of the natural joint. Attempts have also been made to develop synthetic lubricants which might be introduced into joints which start to show signs of failure in the same way that an engineer might prolong the life of a bearing in a maehine by introducing an improved lubricant. Finally, and particularly in the case of esteoarthrosis, it may be necessary to consider joint replacement if function has been severely impaired and the patient is subjected to pain. It is important that endoprostheses should be designed on proper engineering principles, otherwise undue wear or loosening will occur. In this article a brief introduction will be given to studies in each of these fields.

\section{Modes of Lubrication}

In engineering situations lubricated bearing behaviour may be described by one of the following regimes: fluid-film lubrication; mixed lubrication; boundary lubrication.

\section{Fluid-Film Lubrication}

This occurs whenever the bearing surfaces are separated by a layer of fluid which is thicker than the sum of th surface roughnesses. The resistance to motion arises $(\oplus)$ entirely from the shearing of the viscous layer, and in general the friction force can be represented by an cquation of the form:

$$
\mathrm{F}=\eta \frac{\mathrm{U}}{\mathrm{h}} \mathbf{A}
$$

where

$$
\begin{aligned}
& \mathrm{F}=\text { fraction force; } \\
& \mathrm{U}=\text { relative sliding velocity; } \\
& \mathrm{h}=\text { lubricant film thickness; } \\
& \mathrm{A}=\text { effective area of bearing; } \\
& \eta=\text { coefficient of viscosity. }
\end{aligned}
$$

The coefficient of viscosity is the most important pro. perty of a lubricant in fluid-film bearings since it governs not only the resistance to motion but also the ability of the bearing to develop adequate load-bearing pressures in the fluid.

The lubrication films in bearings are themselves quite thin in physical terms, normally between $10^{-5}$ and $10^{-3}$ in. This magnitude is normally adequate to separate the opposing bearing surfaces which are, of course, manufactured with a high degree of precision. Surface roughnesses of typical engineering bearings usually range frop one to a few tens of millionths of an ir.ch.

If the apposing bearing surfaces can be prevented from touching each other by interposing a layer of fluid, wear is almost totally prevented and the resistance to sliding is low. It is for this reason that 'fluid-film" is often described as the ideal mode of lubrication. If the loadcarrying pressures are generated by the sliding motion of the surfaces the mechanism is known as the "hydrodynamic' lubrication, but if the pressure is generated in a pump outside the bearing the action is known as 'hydrostatic lubrication'.

The thickness of the fluid layer in a hydrodynamic bearing in which the viscosity of the fluid remains constant increases as the sliding speed (U) increases and decreases as the load (W) increases, or

$$
\mathrm{h}_{\alpha}\left(\frac{\mathrm{U}}{\mathrm{W}}\right)^{\mathrm{n}}
$$

Another very important feature of hydrodynamic bearing is that the film thickness decreases in the direction, of sliding-a concept knowr as the 'physical wedge' arrangement. 


\section{Boundary Lubrication}

It is clear from equation (2) that the thickness of the lubricant layer will decrease at low speeds and high loads and in due course the opposing bearing surfaces will come into contact at high spots or asperitics. As the will cority of this condition increases the effectiveness of the lubricant layer diminishes, friction rises, and the the lung surfaces start to wear.

It is found that friction and resistance to wear are no Ionger governed by the viscosity of the bulk fluid but by the p-operties of thin surface layer's formed by physical and chemical action on the surfaces of the solids; hence the term 'boundary lubrication'.

If the operating conditions in machinery are severe, as they are in gear boxes and many bearings, steps have to be taken to provide adequate protection for the surfaces under cosditions of boundary lubrication. This is normally achieved in engineering situations by introducing an additive into the bulk lubricant. Substances like fatty acids are frequently used for moderately severe ronditions, whilst extreme pressure (E.P.) additives which act chemically with the bearing solids are employed in hore extreme conditioss.

The resistance to motion under boundary-lubricated conditions is found to obey similar laws to those of dry friction, although the coefficient of friction is normally higher in the latter case. Thus:

$$
\begin{aligned}
\mathrm{F} & =\mu \mathrm{W}- \\
\text { whare } & \\
\mathrm{F} & =\text { friction force; } \\
\mathrm{W} & =\text { applied load; } \\
\mu & =\text { coefficient of friction. }
\end{aligned}
$$

It should be noted from equations (1) and (3) that the friction characteristics of 'fluid-film' and 'boundarylubricated' bearings are quite different.

\section{Mixed Lubrication}

Many bearings operate in the intermediate condition between boundary and fluid-film lubrication. The surfaces are not completely separated by a layer of fluid, although the regions of close approach and asperity interaction occur over a very small fraction of the effective bearing area. The friction characteristics of the bearing show indications of both mechanisms, and the complete behaviour is thus known as 'mixed lubrication'.

\section{riction}

Under conditions of dry contact or boundary lubricat.on equation (3) gives a good description of the resistance to sliding motion. This equation reflects the wellknown laws of friction, known as Amontons' Laws, which state that:

1. the force of friction is independent of the apparent bearing area;

2. the force of friction is directly proportional to the applied load.

Most surfaces are quite rough on a molecular scale and when two solids come together they touch only at high points (or asperities). Asperity interactions cause appreciable local elastic and plastic deformation but the real area of contact normally remains a very small fraction of the apparent bearing area. It is, of course, the real contact area which governs the resistance to sliding, and this explains Amontons' first finding that the force of friction is independent of the apparent bearing area. The local shear stresses on the areas of asperity interaction are found to be reasonably uniform, and since the real contact area is directly proportional to total oad (W); thus explaining Amontons' second law.

\section{Wear}

Although practically everything which an engineer makes will ultimately wear out, this aspect of tribology is the least explored. The physical and chemical actions involved in the wear process are complex, and much current research work is devoted to their elucidation. Well-known forms of wear include abrasion, adhesion, fatigue, erosion and corrosion.

Abrasive wear may occur when a rough, hard surface slides over a softer one (e.g. steel over plastic), or when hard particles are trapped between rubbing surfaces.

In the latter case the abrasive material may enter the conjunction from the environment, or it may be debris resulting from the wear process.

Adhesive wear, which occurs as a result of local welding at asperity junctions, is by far the most common wear mechanism. In many cases the volume of material removed in the adhesive wear process satisfies the following relationships:

where

$$
\mathrm{V}=\mathrm{k} \frac{\mathrm{Wx}}{3 \mathrm{p}_{\mathrm{m}}}
$$

$$
\begin{aligned}
& \mathrm{V}=\text { volume of material worn away; } \\
& \mathrm{W}=\text { applied load; } \\
& \mathrm{x}=\text { total sliding distance; } \\
& \mathrm{p}_{\mathrm{m}}=\text { hardness of softer material; } \\
& \mathrm{k}=\text { wear coefficient; }
\end{aligned}
$$

This equation enables the bearing designer to estimate the useful life of a bearing or endoprosthesis under given operating conditions. It also enables the wear resistance of different materials to be evaluated if the hardness can be measured and the dimensionless wear coefficient evaluated.

\section{Synovial Joints}

The synovial joint possesses many of the features of engineering bearings. The bearing inaterial 'articular cartilage' is supported on a relatively hard backing (bone) and lubricated by a viscous fluid (synovial fluid). It is necessary to understand the properties of all these bearing elements and the conditions encountered in the joint if the lubrication mechanism is to be resolved.

Articular cartilage is a relatively soft, porous material with a surcace roughness about ien times greater than conventional engineering bearing surfaces. It deforms under load and the fluid contained within the porous material is slowly expelled. Synovial fluid is a dialysate of blood plasma to which is added hyaluronic acid, manufactured by the synovial membrane. The fluid is non-Newtonian as far as its viscous properties are concerned.

Thus at low shear rates when the joint is almost stationary, synovial fluid is usually at least as viscous as glycerine. At the highest shear rates encountered in running it is only a few times more viscous than water. The additive mainly responsible for the viscous property of synovial fluid is hyaluronic acid. Nature has provided a fluid which gives excellent tribological properties for the conditions encountered in the human joint.

Synovial joints are really dynamically loaded bearings. In the case of the hip and knee joints the loads vary considerably. 1t has been shown that in the normal walking cycle loads on the his joint can rise to three or four times bodyweight. Similar loads are encountered in the knee. In vigorous exercises or accidents much greater forces may be applied to the articular surfaces.

From a knowledge of the overall geometry of synovial joints, the properties of articular cartilage and synovial fluid, the sliding speeds, and loads encountered in typical activities, it is possible to estimate the thickness of the 
effective lubricating layer. Several important results emerge from such investigations, but the main conclusions are as follows:

1. During exercises like walking the effective layer of lubricant is thicker than the surface roughness of healthy articular cartilage (even though the latter is rough compared with engineering bearing surfaces): i.e. fluid-film lubrication exists for part of the cycle.

2. The local deformation of the elastic cartilage encourages the generation of this effective layer of lubricant during sliding motion and the joint thus experiences elastohydrodynamic lubrication.

Newtonian behaviour) leads to enhanced protection

3. At heel-strike the load rises considerably and the joint surfaces move towards each other to give a form of fluid-film lubrication known as squeeze-film action, particularly when sliding velocities are low.

4. During squeeze-film action the unique combination of cartilage properties (particularly elasticity, porosity, and surface quality) with synovial fluid characteristics (mainly molecular composition and nonNewtonian behaviour) leads to enhanced protection for the articular surfaces. This has been termed 'boosted lubrication'.

5. After prolonged loading and possibly at the time of 'toe-off', when the second loading peak is applied to the joint in the walking cycle, articular surfaces come very close together and are protected by boundary lubrication action.

It is clear that no unique mode of lubrication governs the behaviour of load-bearing synovial joints. Indeed, the joint appears to encounter all the conventional lubrication mechanisms known to the engineer. In addition, the remarkable ability of the joint to resist squeeze-film action appears to be unique to the biological system.

\section{Synthetic Lubricants}

The possibility of influencing the development of osteoarthrosis by introducing a synthetic lubricant into the capsule of a joint has been explored in recent years. Lubricants which resemble synovial fluid in flow terms have been subjected to toxicity tests and a number of clinical trials have been undertaken. Silicones and polymer solutions have been evaluated, and although some patients demonstrated remarkable improvement, there is not sufficient evidence to promote confidence in the view that lubricant changes in human ijoints will soon be commonplace. Extensive studies of potential lubricants leading to clinical trials are now in hand and it will be some time before the full potential of this approach can be evaluated.

\section{The Behaviour of Replacement Joints}

Two of the major problems encountered in endo. prosthetic design are tribological in nature. In the first place, the rate of wear has to be sufficiently low to enable the joint to operate satisfactorily as a bearing for the remaining life of the human machine. Secondly, the friction force developed between the sliding surfaces must be minimised, not only to provide freedom of movement for the patient, but also to limit the severity of the problem of implant fixation.

Most replacement joints are either of the metal-on. metal or metal-on-plastic varieties. If two metals are used it is customary to employ like materials to avoid corrosion problems in the hostile environment of the body. Such a combination of metals normally leads to high friction and is generally avoided in engineering bearings. In the human joint many successful metal-onmetal bearings based upon chrome-cobalt alloys have been designed and used. They have a relatively 16 wear-rate but the friction forces and torques are high compared with metal-on-plastic bearings. The main wear mechanisms encountered in metal-on-metal joints are abrasion and adhesion.

In recent years designers have turned increasingly to metal-on-plastic combinations of materials. Stainless steels and chrome-cobalt alloys have been used and the most popular plastic has been medium-density, highmolecular weight polyethylene. These combinations provide much lower friction, thus relieving the fixation problem, but the plastic tends to wear away by adhesion. abrasion, and possibly fatigue. Careful laboratory studies have shown that in load-bearing joints such as the hip and the knee the wear rate is sufficiently low to enable useful lives to some twenty-five years to be envisaged for this kind of joint.

The search for improved bearing materials for endoprosthetic development continues: alongside fundamental studies of the friction and wear process. Replacement joints usually operate in the presence of a fluid similar to healthy synovial fluid, but it is important to realise that those joints made of a metal-on-plastic combination cf materials could perform adequately as dry bearings.

Replacement human bearings are now available in a variety of forms for the hip, knee, shoulder, elbow and finger. Development has been rapid during the past decade and there appears to be every prospect th steady and possibly spectacular progress will be made the next ten years.

Article received August, 1976 